\title{
New developments in clinical aspects of lymphatic disease
}

\author{
Peter S. Mortimer ${ }^{1}$ and Stanley G. Rockson ${ }^{2}$
}

${ }^{1}$ St. George's Hospital Medical School, University of London, London, United Kingdom. ${ }^{2}$ Stanford University School of Medicine, Stanford, California, USA.

\begin{abstract}
The lymphatic system is fundamentally important to cardiovascular disease, infection and immunity, cancer, and probably obesity - the four major challenges in healthcare in the 21st century. This Review will consider the manner in which new knowledge of lymphatic genes and molecular mechanisms has demonstrated that lymphatic dysfunction should no longer be considered a passive bystander in disease but rather an active player in many pathological processes and, therefore, a genuine target for future therapeutic developments. The specific roles of the lymphatic system in edema, genetic aspects of primary lymphedema, infection (cellulitis/erysipelas), Crohn's disease, obesity, cancer, and cancer-related lymphedema are highlighted.
\end{abstract}

The lymphatic system is arguably the most neglected bodily system, and as a result, its contribution to human health and disease is not well understood. As Philip McMaster said in his Harvey Lecture of 1942, "The functions of the lymphatic system have remained largely unknown (since its discovery). Two influences have contributed to our state of ignorance, a lack of suitable methods of study ... and a lack of interest" (1). Not much changed until the era of molecular science and medicine. In the time since McMaster's lecture, genes and molecular proteins specific to the lymphatic system have been discovered, which has enabled a greater understanding of lymphatic development and the active role of lymphatics in cellular and physiological processes.

The lymphatic system has three major functions. The first is the preservation of fluid balance; the second is a nutritional function, as intestinal lymphatics are responsible for fat absorption; and the third function is host defense.

Lymph vessels return the capillary ultrafiltrate and escaped plasma proteins from most tissues back (ultimately) to the blood circulation. Working in partnership with the cardiovascular system, the lymphatics are responsible for maintaining tissue (and plasma) volume homeostasis. Impaired lymph drainage results in peripheral edema (lymphedema) and may have more far-reaching effects on cardiovascular disease, in particular hypertension and atherosclerosis.

Lymphatics have an important immune surveillance function, as they represent the principal route of transport from tissues for antigen and immune cells. As such, lymphatics are important for adaptive immunity. Impaired lymphatic function predisposes to infection, which can clinically manifest as cellulitis/erysipelas, one of the most common medical conditions to present to hospital emergency departments. Furthermore, lymphatics appear to be important for self-tolerance. A failure to suppress the immune response to cleared peripheral tissue antigen(s) can result in autoimmune disease.

Intestinal lymphatics (lacteals) are responsible for most fat absorption, first documented by Gaspare Aselli in 1627, when the lymphatic system was discovered (2). A relationship between fat and lymphatics may exist well beyond the gut alone. Fat deposition is a defining clinical characteristic of lymphedema. Suction-assisted lipectomy

Conflict of interest: Peter S. Mortimer has received in excess of $\$ 50,000$ from Cancer Research UK and the British Heart Foundation.

Citation for this article: J Clin Invest. 2014;124(3):915-921. doi:10.1172/JCI71608. of lymphedema has shown that the swelling is not just fluid, but is dominated by fat (3). The lymphatics serve as the main pathways for the removal of inorganic material (e.g., silica and carbon) as well as dying and mutant cells. The lymphatic vasculature and nodal tissue is the preferred route for the metastatic spread of cancer. Accordingly, the lymphatic system may be important for defense against cancer by generating immune responses to malignant cell antigens (4). The prevention of the lymphatic entry and propagation of malignant metastasis would effectively render the cancer nonfatal.

As one can see, the lymphatic circulation is fundamentally important to cardiovascular disease, infection and immunity, cancer, and in all likelihood, obesity - the four major challenges to healthcare in the 21 st century.

\section{When is peripheral edema considered to be lymphedema?}

Edema is the presence of an excess of interstitial fluid and is an important sign of ill health in clinical medicine. It may occur in the lungs (pulmonary edema), the abdominal cavity (ascites), and other body cavities (synovial, pericardial, and pleural effusions), but the most common site is within the peripheral subcutaneous space.

In medical practice, peripheral edema is often classified according to possible systemic causes, such as heart failure, nephrotic syndrome, and venous obstruction. This clinical approach fails to appreciate (a) that more than one cause may contribute to development of the edema and (b) the central role of lymphatic drainage in tissue fluid balance. Consequently, the clinician's approach to treating chronic edema is often misguided and inappropriate as, for example, when diuretics are empirically prescribed.

Edema develops when the microvascular (capillary and venular) filtration rate exceeds lymph drainage for a sufficient period because the microvascular filtration rate is high, lymph flow is low, or a combination of the two. Filtration rate is governed by the Starling principle of fluid exchange. In simple terms, microvascular filtration of fluid from capillary into interstitium is driven by the hydraulic (water) pressure gradient across the blood vessel wall $\left(P_{c}-P_{i}\right.$, in which $P_{c}$ indicates capillary pressure and $P_{i}$ indicates interstitial pressure) and is opposed by the osmotic pressure gradient $\left(\pi_{p}-\pi_{I}\right.$, in which $\pi_{p}$ indicates plasma osmotic pressure and $\pi_{i}$ indicates interstitial osmotic pressure from tissue proteins), which is the suction force retaining fluid within the vessel. 
A

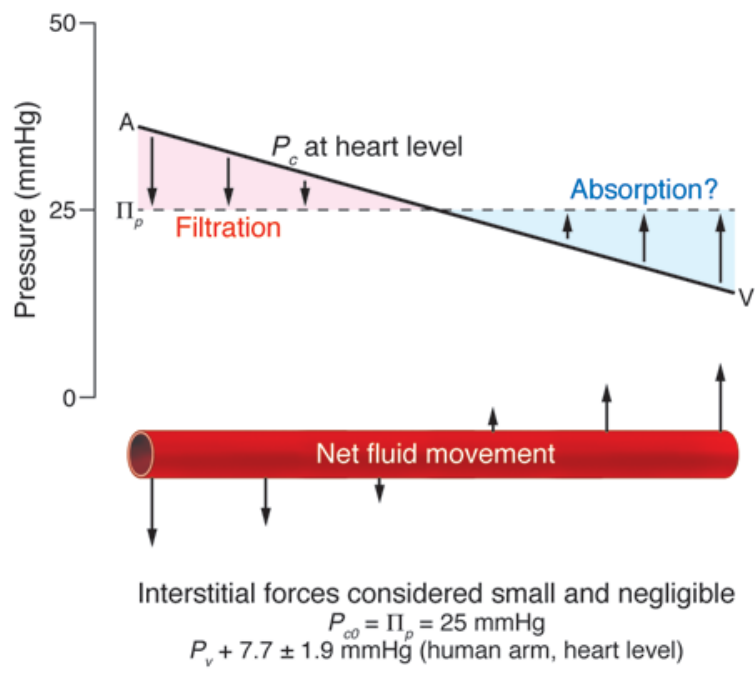

B

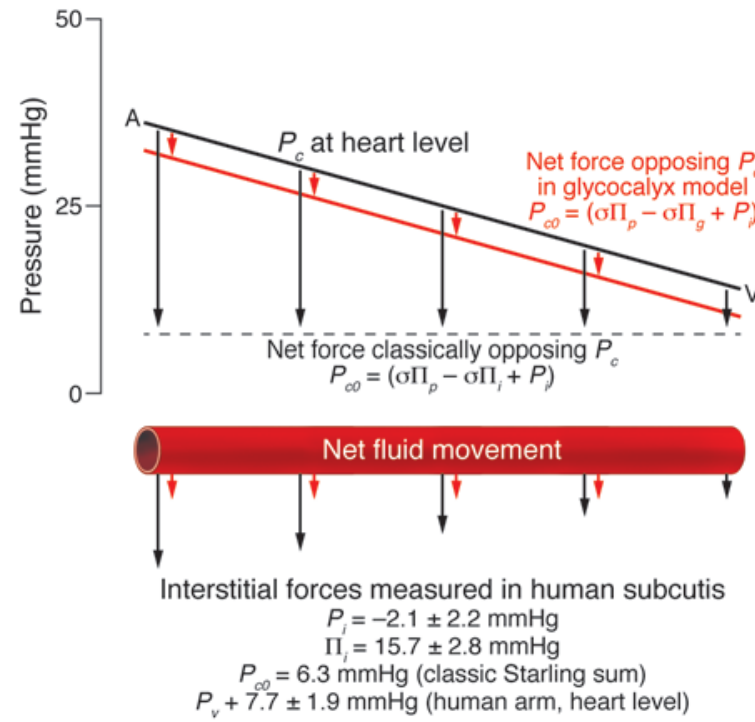

\section{Figure 1}

The traditional view of the lymphatic capillaries is that the arteriolar end of the capillary filters, while the venular end of the capillary reabsorbs fluid. However, if all Starling forces are considered, including interstitial colloid osmotic and hydraulic pressures, there is a net but dwindling filtration along the entire length of the capillary, with no venous reabsorption. $(\mathbf{A})$ In the traditional view, interstitial forces are deemed negligible (the sum of Starling forces opposing filtration $\left[P_{0}\right]=25 \mathrm{mmHg}, P_{V}=7.7 \mathrm{mmHg}$ ). (B) However, interstitial forces are taken into account when using direct measurements $\left(P_{i}=-2.1 \mathrm{mmHg}, \pi_{i}=15.7 \mathrm{mmHg}, P_{0}=6.3 \mathrm{mmHg}, P_{v}=7.7 \mathrm{mmHg}\right)$. Adapted with permission from Cardiovascular Research (6).

Accumulation of capillary filtrate in the tissue spaces is avoided mainly through lymph drainage and not, as was previously thought, through reabsorption. Traditionally (and still often explained incorrectly in most undergraduate medical school teaching manuals and books), it was taught that $90 \%$ of interstitial fluid is reabsorbed at the venous end of the capillary. This view is not supported by modern evidence, which demonstrates that in most vascular beds, there is a net but dwindling filtration along the entire length of well-perfused capillaries. When measurements are made directly of $P_{i}$ and $\pi_{i}$ in muscle, mesentery, or warm skin at the cardiac level, the sum of all Starling forces is not an absorptive force in the venous capillaries, but rather a slight filtration force. Data from 12 tissues confirm that venular blood pressure exceeds the sum of pressures opposing filtration in the steady state (5). Exchange vessels (mainly capillaries and post-capillary venules) can resorb fluid for a short period if the Starling pressures are disturbed (e.g., hemorrhage will drop $P_{c}$ sufficiently to allow transient venous reabsorption), but the Starling forces quickly readjust to a state of filtration (Figure 1).

The reason for non-sustained microvascular reabsorption is that (a) ultrafiltration of the absorbed interstitial fluid at the underside of the glycocalyx (of the blood capillary wall) raises subglycocalyx plasma protein concentration $\left(\pi_{g}\right.$ and $\left.\pi_{i}\right)$, and (b) $P_{i}$ falls as fluid is removed from the interstitium. The changes in $\pi_{g}$ and $P_{i}$ gradually abolish the net absorptive force so that, ultimately, a steady state of slight filtration is restored (6).

Tissue fluid balance thus depends critically upon lymphatic function in most tissues. By returning the capillary ultrafiltrate and filtered plasma proteins to the bloodstream, lymphatic vessels complete the extravascular circulation of fluid and protein and maintain tissue and, to some extent, plasma volume homeostasis. If lymphatic drainage fails to cope with the excessive microvascu- lar filtration caused by increased $P_{c}$ (e.g., heart failure, postphlebitic syndrome), or with reduced plasma colloid osmotic pressure (e.g., nephrotic syndrome, malnutrition, liver disease, or increased endothelial conductance with reduced reflection coefficient, as in inflammation), then a filtration edema occurs.

All chronic edema indicates an inadequacy or failure of lymph drainage; therefore, a clinical approach to peripheral (i.e., subcutaneous) edema should begin with a consideration of lymphatic function to assess whether this is a primary impairment or whether a normal lymphatic circulation is simply overloaded by high microvascular filtration. There are many clinical circumstances in which more than one physiological factor contributes to chronic peripheral edema. For example, in heart failure, edema is caused initially by high microvascular filtration from high venous pressures that overwhelms lymph drainage capacity. However, with chronicity, the sustained microvascular filtration exhausts lymph drainage so that, eventually, even when heart failure is controlled, permanent damage to lymphatic vessels results in lymphedema. Therefore, a clinical approach should consider all possible physiologic factors influencing both lymph drainage and microvascular filtration, rather than relying upon a diagnosis confined to a single clinical category such as heart failure. Arguably, it may be better to consider the presence of chronic edema as synonymous with the presence of lymphedema, inasmuch as all edema represents relative lymph drainage failure.

\section{Impaired lymph drainage and pathological mechanisms}

The evidence supporting the mechanisms for the entry of interstitial fluid into and transport along lymphatic vessels in humans remains unclear. The absorbing initial lymphatic network is usually a blind-ended system of lymphatic capillaries. The wall of a lymphatic capillary has no smooth muscle invest- 


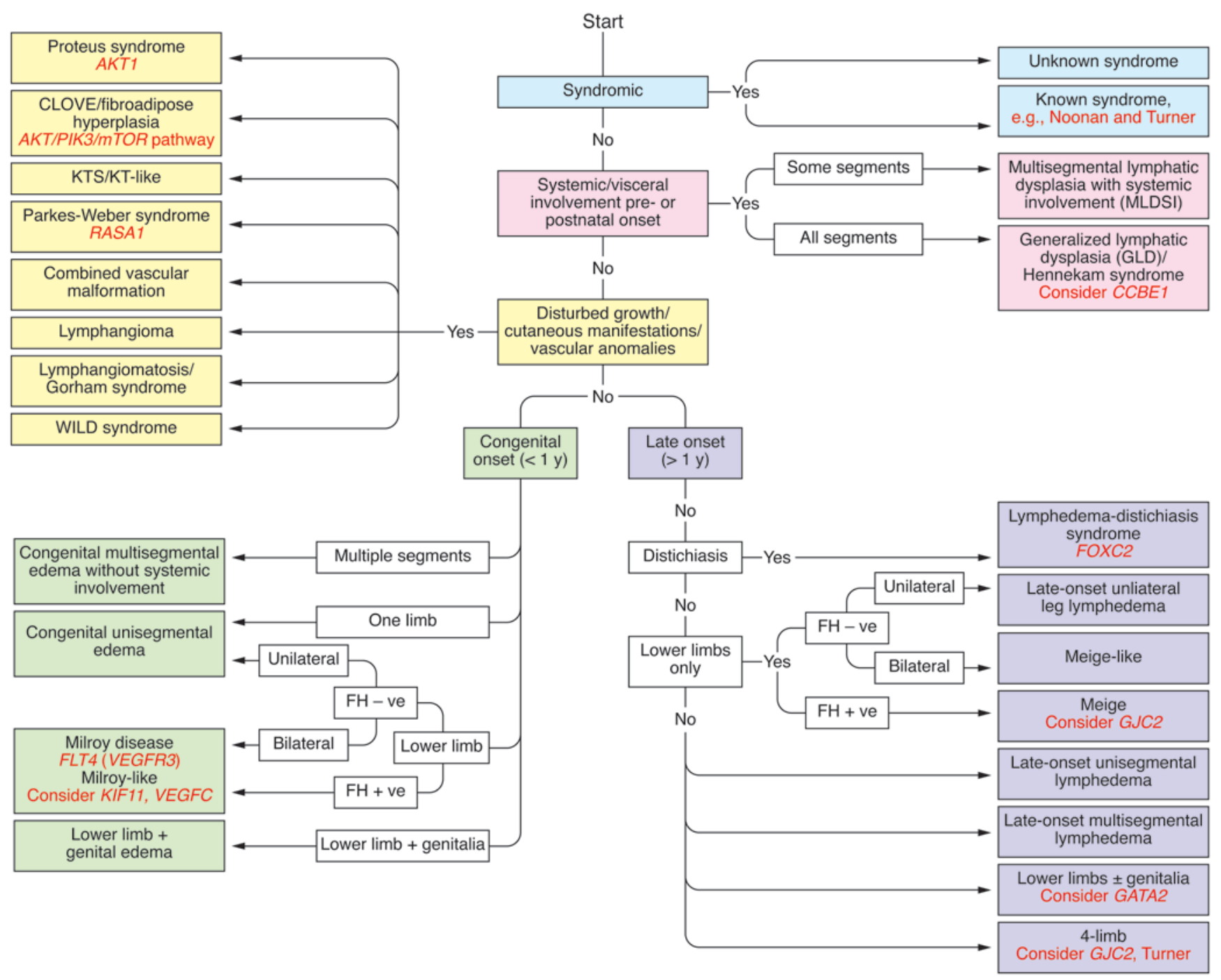

Figure 2

Clinical algorithm for classification of primary lymphoedema according to genotype and, where the gene is not known, phenotypic cohort. Adapted with permission from Clinical Genetics (24). FH, family history.

ment and therefore does not contract. Some of the endothelial intercellular junctions (flap valves) are at least $14 \mathrm{~nm}$ in diameter, making it possible for particulate matter, and even aggregates of cancer cells, to enter. When the interstitial pressure exceeds the intraluminal pressure, these junctions open, allowing inflow of fluid until pressures equilibrate, whereupon the flap valves close. The interstitial fluid is probably driven into the initial lymphatic by a small hydraulic pressure gradient. Mutations in the VEGFR3 gene cause Milroy disease (7). The mechanism for this form of human lymphedema is not an absence of initial lymphatics, as animal models had suggested, but a failure of initial lymphatic fluid absorption (8). Mutations in the FOXC2 gene cause aberrant smooth muscle surrounding initial capillaries (9) and may contribute to the lymphatic dysfunction seen in lymphedema-distichiasis syndrome.

The initial lymphatic network drains into a series of collecting vessels that possess both semilunar valves and an envelope of smooth muscle. The main mechanism for lymph drainage in collector lymphatics is therefore lymphatic pumping, with valves to prevent reflux. FOXC2 mutations cause lymphatic valve dysfunction and, consequently, lymph reflux, providing one of the main mechanisms for the development of lymphedema in lymphedema-distichiasis syndrome (9).

Contraction of the lymphatic collectors is initiated by an action potential complex. Efficient pumping depends on the propagation of the electrical impulse that is under the control of pacemaker cells. As with cardiac muscle, which is very similar to lymphatic smooth muscle, calcium influx is important for the pacemaker potential and, therefore, calcium channel antagonists may well cause peripheral edema by interfering with lymphatic pumping (10).

\section{Genetic aspects of primary lymphedema}

Primary lymphedema arises due to an intrinsic, presumably genetic, fault in lymph drainage, whereas the term secondary lymphedema identifies a specific external stimulus or agent that has damaged lymph-conducting pathways (e.g., trauma or surgery). 


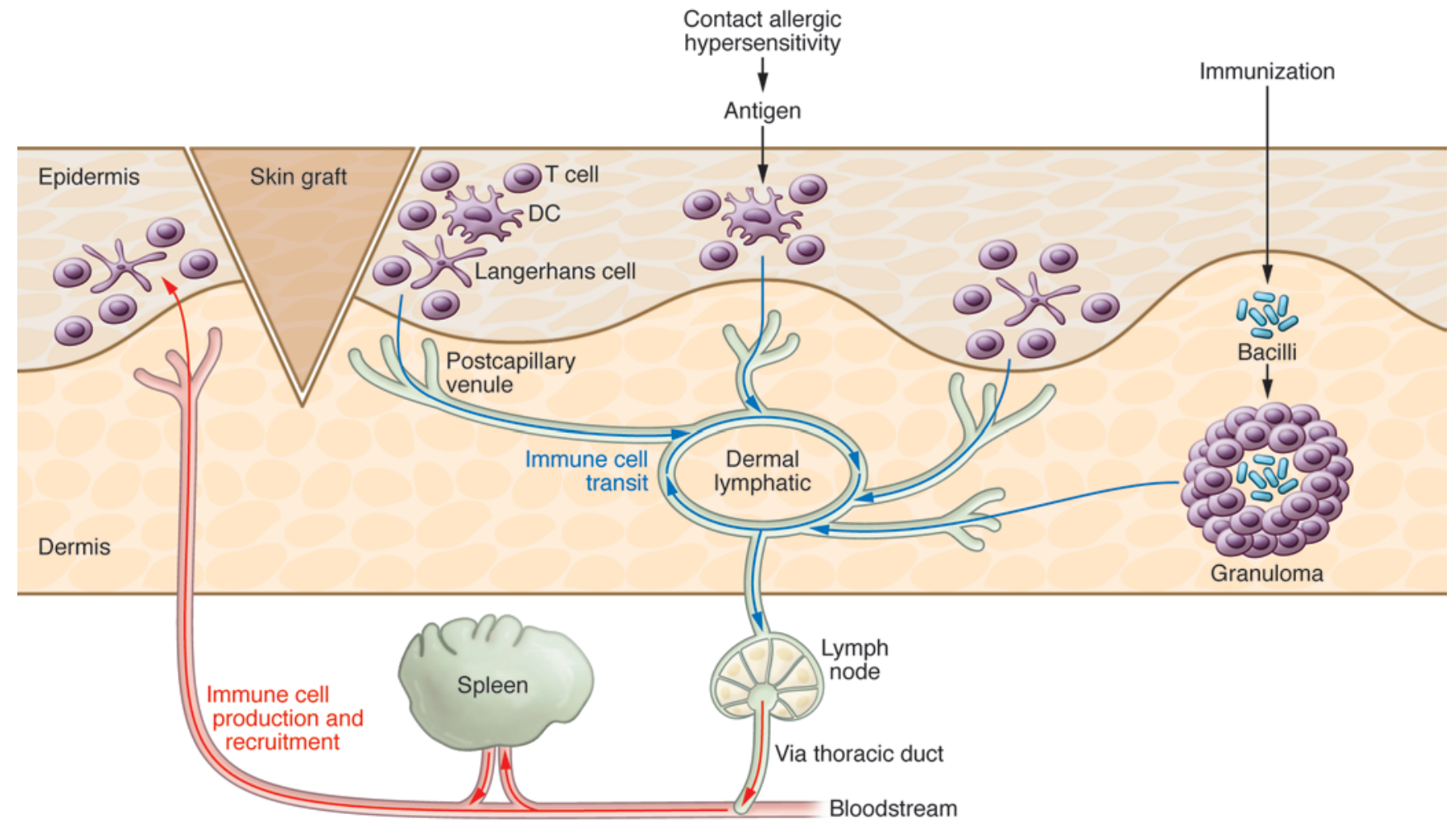

\section{Figure 3}

The dermal lymphatic sits center stage for the drainage of immune-active cells. Skin grafts, allergens, or immunization stimulate a variety of immune cells, including T cells, Langerhans cells, dendritic cells, and macrophages, which exit from the skin via the lymphatic system. Antigenpresenting cells cells exit the dermis via the lymphatic postcapillary venules and travel to the dermal lymphatic and then on to the lymph node, in which they activate and expand lymphocytes that secrete antibodies or otherwise target the antigen. Immune cells then travel through the lymphatic vasculature and enter the blood circulation via the thoracic duct. The blood is filtered through the spleen, which can lead to additional activation and expansion of antigen-targeted immune cells.

Primary lymphedema is caused by faulty lymphatic vascular development, giving rise to either a structural and/or functional abnormality that impairs drainage.

Until recently, the nature and cause of primary lymphedema has remained unknown. Consequently, patients were classified according to clinical features such as age of onset, categorized as congenital (at or shortly after birth), praecox (occurring during an early stage of life, most commonly during the teenage years or early adulthood), and tarda (late in life). The development of direct contrast lymphography helped to distinguish different categories on the basis of structural abnormalities, but fundamental causes remained unknown (11).

The identification of causal mutations in human lymphedema has enabled a better understanding of the role of these genes and their encoded proteins. Although the blood, vascular, and lymphatic systems are structurally and embryologically related and do function in concert, they develop through fundamentally different molecular mechanisms. Nevertheless, the malfunction of lymphatic genes frequently has cardiovascular consequences. For example, mutations in FOXC2, which are causal for lymphedema-distichiasis syndrome, result in congenital heart disease in $5 \%$ of patients.

The first gene identified as causative for human lymphedema was VEGFR3, in 1998 (7, 12). This gene was observed to be mutated in some cases of Milroy disease, a congenital-onset form of lymphedema with swelling of the feet and lower limbs. Hydrocele is seen in one third of affected males, suggesting that lymphatic dysfunction may be implicated in this condition even when unrelated to lymphedema (13). Soon after, the gene for lymphedemadistichiasis syndrome was mapped and subsequently identified as the developmentally important transcription factor FOXC2 (14). Lymphedema-distichiasis syndrome is a syndromic condition in which distichiasis (aberrant eyelashes originating from the meibomian glands) occurs at birth. Onset of lymphedema, aberrant eyelashes originating from a failure of meibomian gland development, however, is delayed until at least puberty (originally classified as praecox), but the presentation can be delayed to as late as the 5 th decade (15). This indicates that genetic forms of lymphedema can present late in life. One striking feature of VEGFR3 and FOXC2 mutations is the very high incidence of venous valve failure. This presumably reflects the fact that, in development, lymphatic endothelial cells derive from venous endothelial cells. FOXC2 mutations cause superficial vein incompetence in $100 \%$ of cases and deep vein incompetence in up to one third of cases (16).

Identification of additional genes occurred slowly. The extremely rare syndrome hypotrichosis-lymphedema-telangiectasia syndrome was shown in 2003 to be caused by mutations in SOX18 (17), while in 2009 CCBE1 was identified as the gene underlying autosomal recessive generalized Hennekam lymphangiectasia-lymphedema 
syndrome (18). Recently the gene GJC2 was discovered (19). GJC2 encodes connexin 47 and appears to affect gap junctions. The predominant phenotype for mutations of this gene is late-onset fourlimb involvement, but there is a wide diversity in age of onset and extent of limb involvement (20). Additional genes, very recently identified, are GATA2 (21) and KIF11 (22). GATA2 causes Emberger syndrome and is interesting because of the relationship between lymphedema, immunodeficiency, and myelodysplasia, the latter predisposing to acute myeloid leukemia. Mutations in KIF11 cause microcephaly-lymphedema syndrome, for which the lymphedema phenotype is identical to Milroy disease, with congenital onset and restriction of distribution to feet and legs. Recently a mutation in VEGFC was found to be causal for Milroy disease, in which no mutations were found in VEGFR3 (23). To date there are at least 9 causal mutations known for inherited human lymphedema (Figure 2).

As causal genes are identified, the possibility arises for a classification built on patient phenotypes for which the gene is known. This work is in progress, but is providing a more structured clinical approach than simply relying on age of onset alone (ref. 24 and Figure 2).

\section{Immunity, inflammation, and infection}

One of the lymphatic system's main functions is in host defense. Everyone has experienced enlarged, tender lymph nodes in the neck (lymphadenitis) in response to a throat infection. The afferent lymphatic vasculature provides the major exit route from the tissues for soluble antigens and for immunologically active cells (e.g., lymphocytes, dendritic cells, and macrophages) (Figure 3).

Without intact lymphatics a primary immune (adaptive) response cannot occur. Their importance was first realized in the early days of tissue transplantation, when interruption of lymph drainage pathways were found to delay rejection of skin allografts (25).

The lymph node exists to filter the lymph that passes through it, to process antigens, and to produce an appropriate immune response. Afferent lymph is important as a stimulus to lymph node structure and function. Involution of deafferentized lymph nodes is poorly understood (26).

Recurrent infection (e.g., interdigital fungal infection or cellulitis/ erysipelas) is a common event in lymphedema. Cellulitis is an acute spreading inflammation of the skin and subcutaneous tissues caused by bacteria, usually Streptococcus or Staphylococcus. It is likely that disturbances in immune cell trafficking compromise tissue immunosurveillance, but the exact mechanism is not known. Cellulitis can develop for the first time as a result of subclinical, and therefore previously unsuspected, lymph drainage abnormalities (27). Lymphedema conveys a 71-fold enhancement of risk for cellulitis, relative to normal conditions (28). In a community study of 221 patients with lymphedema in South West London followed prospectively for 12 months, 65 (29\%) suffered at least one attack of cellulitis and 16 of those required hospital admission with a mean length of stay of 12 days. Therefore, cellulitis arising on a background of disturbed lymph drainage is a common and expensive healthcare problem (29).

Infections are common to all forms of lymphedema, without regard to etiopathogenesis. In filariasis, progression of lymphedema, and therefore elephantiasis (the gross tissue changes seen in advanced lymphedema giving rise to skin resembling elephant hide), is considered a two-step process - the first initiated by the filarial parasite and host innate immune system and the second propagated by the host's adaptive immune system and secondary infection (30). With an estimated 120 million people infected by lymph-dwelling filarial parasites, 40 million have lymphedema and secondary infections, thereby creating an enormous global disease burden.

Primary lymphedema for which the causal gene is known (e.g., Milroy lymphedema due to mutations in VEGFR3) is associated with increased rates of infection, but only in the affected lymph drainage basin, i.e., feet and legs. Bacterial (cellulitis), fungal (athlete's foot), and viral (human papillomavirus wart) infections are all increased in prevalence (10). Studies in the K14-VEGFR-3-Ig mouse have helped to explain the reasons for such infections. These studies have demonstrated the importance of lymph drainage to the organization and function of B cells resident in the draining lymph nodes and to the generation of an appropriate humoral response, with efficient antibody production. Another interesting finding is that of impaired cellular tolerance to peripheral tissue antigens; this may have implications for the development of autoimmune disease (31).

In most cases of lymphedema, systemic immunity is not recognizably altered. However, in generalized lymphatic dysplasia (Hennekam syndrome) caused by mutations in the CCBE1 gene, disturbances in lymphocyte subsets and plasma immunoglobulins can be detected (personal observation). To what extent immunological abnormalities reflect problems of lymphoid development (synthesis) or loss due to either the presence of intestinal lymphangiectasia or a failure of lymphocyte homeostasis remains to be delineated. Haploinsufficiency for GATA2 causes Emberger syndrome, comprising human immunodeficiency and lymphedema as well as myelodysplasia progressing to myeloid leukemia. Clinically this can manifest with extensive cutaneous human papilloma virus infections. Therefore, an association between altered systemic immunity and lymphedema can occur (32). Lymphedema associated with immunodeficiency can also occur with NEMO mutations (33).

The range of diseases associated with lymphatic dysfunction causing disturbed immunity is likely to be extensive. An example is inflammatory bowel disease, particularly Crohn's disease (CD). CD is a chronic relapsing inflammatory disease of the gastrointestinal tract. Abnormalities in lymphatic drainage have been noted in the original descriptions of CD but essentially ignored since the original publication (34). There is increasing evidence that affected patients share a common phenotype, namely impaired immune responses to bacterial infection. Genetic and genomic studies point to immune deficiency, rather than autoimmunity, as the functional lesion in CD. It is postulated that the dysregulated macrophage response to bacteria is central to the pathogenesis of CD (35). Given the importance of the lymphatic system to innate immunity and macrophage function, it is not difficult to hypothesize a role for lymphatic dysfunction in CD. This hypothesis is clinically supported by the development of genital lymphedema caused by granulomatous inflammation infiltrating the skin (and dermal lymphatics) in the anogenital region (anogenital granulomatosis) (36). Recurrent attacks of inflammation resembling cellulitis occur, and up to one third of patients affected are found to have gastrointestinal CD.

\section{Fat and lymphatics}

For years, surgeons operating on lymphedema have observed "wet fat," suggesting that the swelling of lymphedema reflects fat deposition and not just the presence of excess fluid. This has led to the development of circumferential suction-assisted lipectomy as a treatment for lymphedema in cases in which fat hypertrophy dominates and fluid is minimal $(37,38)$. 
The absorption of fat by intestinal lacteals and transport by the lymphatic system has been recognized for centuries, but the relationship between peripheral fat and lymphatic function is only just being realized. Peripheral tissue lipid transport and homeostasis may well be in part determined by lymphatic function, hence the increased fat deposition seen in lymphedema. Mice with a heterozygous Prox1-inactivating mutation have leaky lymphatic vessels and develop obesity and inflammation (39). As lymph drainage is responsible for the return of interstitial proteins to the blood, it seems likely that extravascular lipoproteins would follow the same principle (40).

While impaired lymph drainage might influence fat disposition and obesity, so the reverse appears to be likely. Weight gain is a strong risk factor for the development of breast cancer-related lymphedema (BCRL) (41). Using an isotope clearance technique, lymph drainage was found to be significantly lower in obese human subjects when compared with lean controls (42). In a model of hypercholesterolemia, lymphatic function was severely compromised, including impaired dendritic cell migration. Removal of cholesterol from peripheral tissues via reverse cholesterol transport requires lymph drainage (43). This is likely to have implications for the pathogenesis of atherosclerosis; however, the exact mechanisms linking lipid pathologies (obesity, diabetes, hypercholesterolemia, and obesity) with lymphatic function remain to be elucidated.

\section{Cancer}

The preferential route for the spread of cancer cells is via the lymphatic vasculature. This principle underpins the use of sentinel lymph node biopsy in many cancers for the early detection of metastatic spread. Indeed, even hematogenous spread of cancer may require entry into lymphatics first before transfer to the bloodstream via lymphovenous communications. Basic physiological principles teach us that the blood circulation supplies tissues with oxygen, nutrients, and water, while it is the responsibility of the lymphatic vasculature to drain the tissues of proteins, cells, and surplus fluid. Harnessing the migration of cancer cells toward, into, and along initial lymphatics would be a big step in cancer treatment.

There are many possible causes for secondary lymphedema, the most common of which is probably cancer treatment, at least in the developed world. Work in BCRL has demonstrated that obstruction to lymph drainage following removal of axillary lymph nodes is far too simplistic a mechanistic explanation. BCRL develops in women with higher, not lower, peripheral lymph flows following surgery. Furthermore, women who subsequently develop BCRL also have significantly higher lymph flow after surgery in the contralateral arm. This suggests that the development of BCRL is systemically driven and is not solely dependent on changes to outflow resistance in the ipsilateral arm alone (44).

The high prevalence of acquired lymphedema as a sequela of cancer treatment remains problematic. Relative risk for the development of lymphedema is difficult to ascertain, a problem that is compounded by the tendency to protracted periods of latency among these patients $(45,46)$. If one assumes that, in breast cancer, for example, $15 \%-20 \%$ of disease survivors are at clinical risk of lymphedema development (47), how are these individuals to be distinguished from the remaining $80 \%-85 \%$ that will remain free of swelling? The difficulty that surrounds risk stratification and detection of early or subclinical lymphedema is a major impetus for the identification of serologic disease biomarkers. The development of such sensitive and accurate diagnostic modalities would also subserve the growing likelihood that molecular and pharmacologic approaches to therapy might soon be feasible.

Recent preliminary progress has been made in this regard. Based upon the results of transcriptional profiling in a murine model of acquired lymphedema (48), microarray-based transcriptomics of human skin has recently produced an a priori identification of candidate biomarkers for human lymphedema (49). The identified proteins represent four of the central pathogenetic modalities of the disease: lymphangiogenesis, inflammation, fibrosis, and lipid metabolism. Further studies are warranted to determine whether this newly identified biomarker panel will possess utility as an instrument for in vitro diagnosis of early and latent disease.

\section{Future therapeutics}

Current treatments for lymphedema do not reverse the fundamental pathophysiology and therefore provide, at best, stabilization and modest delays in the appearance of end-stage sequelae, such as disfigurement and loss of function. Growing insights into the molecular pathogenesis of both acquired and primary forms of lymphedema provide hope that focused therapeutics might become a reality. As one example, the promise of successful therapeutic lymphangiogenesis has been realized in a variety of animal model applications (50-53). Much work remains to bring these approaches to the human clinical arena. Additionally, enthusiasm for growth factor-mediated therapeutics might be dampened by the cancer-survivor status of a large proportion of the potential recipients (49). Alternative systemic approaches to development of a lymphedema-reversing pharmacology, including targeted antiinflammatory therapy, are beginning to show promise (54-56).

\section{Conclusions}

There are persuasive reasons for believing that the lymphatic system is considerably involved in the four most pressing areas of today's healthcare, namely, cardiovascular disease, cancer, obesity, and infection. Given the absence of any current pharmacological or surgical intervention that can improve lymph flow or lymphatic function, there is an urgent need to redress this failing in medical therapy. The newer developments in lymphatic science raise hopes for meaningful advances in the near future.

Address correspondence to: Peter S. Mortimer, St. George's Hospital Medical School, University of London, London, SW17 ORE, United Kingdom. Phone: 44.0.20.8725.1784; Fax: 44.0.20.8725.5955; E-mail: mortimer@sgul.ac.uk.
1. McMaster PD. Lymphatic participation in cutaneous phenomena. Harvey Lecture, April 16, 1942. Bull N Y Acad Med. 1942;18(11):731-767.

2. Aselli G. De Lactibus Sive Lacteis Venis. Milan, Italy: J.B. Bidellius; 1627.

3. Brorson H, Ohlin K, Olsson G, Nilsson M. Adipose tissue dominates chronic arm lymphedema following breast cancer: an analysis using volume rendered
CT images. Lymphat Res Biol. 2006;4(4):199-210.

4. Turk JL. Delayed Hypersensitivity. Amsterdam: North-Holland Publishing Company; 1967:138.

5. Levick JR. Revision of the Starling Principle: new views of tissue fluid balance. J Physiol. 2004;557(pt 3):704.

6. Levick JR, Michel CC. Microvascular fluid exchange and the revised Starling principle. Cardiovasc Res. 2010;87(2):198-210.
7. Karkkainen MJ, et al. Missense mutations interfere with VEGFR-3 signalling in primary lymphoedema. Nat Genet. 2000;25(2):153-159.

8. Mellor $\mathrm{RH}$, et al. Lymphatic dysfunction, not aplasia, underlies Milroy disease. Microcirculation. 2010;17(4):281-296.

9. Petrova TV, et al. Defective valves and abnormal mural cell recruitment underlie lymphatic vascu- 
lar failure in lymphedema distichiasis. Nat Med. 2004;10(9):974-981.

10. Toland HM, McCloskey KD, Thornbury KD McHale NG, Hollywood MA. Ca(2+)-activated $\mathrm{Cl}(-)$ current in sheep lymphatic smooth muscle. Am J Physiol Cell Physiol. 2000;279(5):C1327-C1335.

11. Browse NL, Stewart G. Lymphoedema: pathophysiology and classification. J Cardiovasc Surg (Torino). 1985;26(2):91-106.

12. Ferrell RE, et al. Hereditary lymphedema: evidence for linkage and genetic heterogeneity. Hum Mol Genet. 1998;7(13):2073-2078.

13. Brice G, et al. Milroy disease and the VEGFR-3 mutation phenotype. J Med Genet. 2005;42(2):98-102.

14. Fang J, et al. Mutations in FOXC2 (MFH-1), a forkhead family transcription factor, are responsible for the hereditary lymphedema-distichiasis syndrome. Am J Hum Genet. 2000;67(6):1382-1388.

15. Brice G, et al. Analysis of the phenotypic abnormalities in lymphoedema-distichiasis syndrome in 74 patients with FOXC2 mutations or linkage to 16q24. J Med Genet. 2002;39(7):478-483.

16. Mellor RH, et al. Mutations in FOXC2 are strongly associated with primary valve failure in veins of the lower limb. Circulation. 2007;115(14):1912-1920.

17. Irrthum A, et al. Mutations in the transcription factor gene SOX18 underlie recessive and dominant forms of hypotrichosis-lymphedema-telangiectasia. Am J Hum Genet. 2003;72(6):1470-1478.

18. Alders $\mathrm{M}$, et al. Mutations in CCBE1 cause generalized lymph vessel dysplasia in humans. Nat Genet. 2009;41(12):1272-1274.

19. Ferrell RE, et al. GJC2 missense mutations cause human lymphedema. Am J Hum Genet. 2010; 86(6):943-948.

20. Ostergaard P, et al. Rapid identification of mutations in GJC2 in primary lymphoedema using whole exome sequencing combined with linkage analysis with delineation of the phenotype. J Med Genet. 2011;48(4):251-255.

21. Ostergaard P, et al. Mutations in GATA2 cause primary lymphedema associated with a predisposition to acute myeloid leukemia (Emberger syndrome). Nat Genet. 2011;43(10):929-931.

22. Ostergaard P, et al. Mutations in KIF11 cause autosomal-dominant microcephaly variably associated with congenital lymphedema and chorioretinopathy. Am J Hum Genet. 2012;90(2):356-362.

23. Gordon K, et al. Mutation in vascular endothelial growth factor-C, a ligand for vascular endothelial growth factor receptor-3, is associated with autosomal dominant Milroy-like primary lymphedema. Circ Res. 2013;112(6):956-960.

24. Connell F, et al. The classification and diagnostic algorithm for primary lymphatic dysplasia: an update from 2010 to include molecular findings. Clin Genet. 2013;84(4):303-314.

25. Barker CF, Billingham RE. The role of afferent lym- phatics in the rejection of skin homografts. J Exp Med. 1968;128(1):197-221.

26. Drayson MT, Ford WL. Afferent lymph and lymph borne cells: their influence on lymph node function. Immunobiology. 1984;168(3-5):362-379.

27. Damstra RJ, van Steensel MA, Boomsma JH, Nelemans P, Veraart JC. Erysipelas as a sign of subclinical primary lymphoedema: a prospective quantitative scintigraphic study of 40 patients with unilateral erysipelas of the leg. BrJ Dermatol. 2008;158(6):1210-1215.

28. Dupuy A, et al. Risk factors for erysipelas of the leg (cellulitis): case-control study. BMJ. 1999; 318(7198):1591-1594.

29. Moffatt CJ, et al. Lymphoedema: an underestimated health care problem. QJM. 2003;96(10):731-738.

30. Babu S, Nutman TB. Immunopathogenesis of lymphatic filarial disease. Semin Immunopathol. 2012;34(6):847-861.

31. Thomas SN, et al. Impaired humoral immunity and tolerance in K14-VEGFR-3-Ig mice that lack dermal lymphatic drainage. J Immunol. 2012;189(5):2181-2190.

32. Johnson KD, et al. CiS-element mutated GATA2dependent immunodeficiency governs hematopoiesis and vascular integrity. J Clin Invest. 2012; 122(10):3692-3704.

33. Mansour $S$, et al. Incontinentia pigmenti in a surviving male is accompanied by hypohidrotic ectodermal dysplasia and recurrent infections. Am JMed Genet. 2001;99(2):172-177.

34. von der Weid PY, Rehal S, Ferraz JG. Role of the lymphatic system in the pathogenesis of Crohn's disease. Curr Opin Gastroenterol. 2011;27(4):335-341.

35. Smith AM, et al. Disordered macrophage cytokine secretion underlies impaired acute inflammation and bacterial clearance in Crohn's disease. J Exp Med. 2009;206(9):1883-1897.

36. Van de Scheur MR, van der Waal RI, van der Waal I, Stoof TJ, van Deventer SJ. Ano-genital granulomatosis: the counterpart of oro-facial granulomatosis. J Eur Acad Dermatol Venereol. 2003;17(2):184-189.

37. Brorson H, Svensson H. Complete reduction of lymphoedema of the arm by liposuction after breast cancer. Scand J Plast Reconstr Surg Hand Surg. 1997;31(2):137-143.

38. Damstra RJ, Voesten HG, Klinkert P, Brorson H. Circumferential suction-assisted lipectomy for lymphoedema after surgery for breast cancer. $\mathrm{Br} \mathrm{J}$ Surg. 2009;96(8):859-864.

39. Harvey NL, et al. Lymphatic vascular defects promoted by Prox 1 haploinsufficiency cause adult-onset obesity. Nat Genet. 2005;37(10):1072-1081.

40. Dixon JB. Lymphatic lipid transport: sewer or subway. Trends Endocrinal Metab. 2010;21(8):480-487.

41. Disipio T, Rye S, Newman S, Hayes S. Incidence of unilateral arm lymphoedema after breast cancer: a systematic review and meta-analysis. Lancet Oncol.
2013;14(6):500-515.

42. Arngrim N, Simonsen L, Holst JJ, Bulow J. Reduced adipose tissue lymphatic drainage of macromolecules in obese subjects: a possible link between obesity and local tissue inflammation. Int J Obes (Lond). 2013;37(5):748-750.

43. Lim HY, et al. Lymphatic vessels are essential for the removal of cholesterol from peripheral tissues by SR-BI-mediated transport of HDL. Cell Metab. 2013;17(5):671-684.

44. Stanton AW, et al. Lymphatic drainage in the muscle and subcutis of the arm after breast cancer treatment. Breast Cancer Res Treat. 2009;117(3):549-557.

45. Herd-Smith A, Russo A, Muraca MG, Del Turco MR, Cardona G. Prognostic factors for lymphedema after primary treatment of breast carcinoma. Cancer. 2001;92(7):1783-1787.

46. Rockson SG. Addressing the unmet needs in lymphedema risk management. Lymphat Res Biol. 2006;4(1):42-46.

47. Rockson SG, Rivera KK. Estimating the population burden of lymphedema. Ann N Y Acad Sci. 2008;1131:147-154.

48. Tabibiazar R, et al. Inflammatory manifestations of experimental lymphatic insufficiency. PLoS Med. 2006;3(7):e254

49. Lin S, et al. Prospective transcriptomic pathway analysis of human lymphatic vascular insufficiency: identification and validation of a circulating biomarker panel. PLoS One. 2012;7(12):e52021.

50. Szuba A, et al. Therapeutic lymphangiogenesis with human recombinant VEGF-C. FASEB J. 2002;16(14):1985-1987.

51. Cheung L, et al. An experimental model for the study of lymphedema and its response to therapeutic lymphangiogenesis. BioDrugs. 2006;20(6):363-370.

52. Yoon YS, et al. VEGF-C gene therapy augments postnatal lymphangiogenesis and ameliorates secondary lymphedema. J Clin Invest. 2003;111(5):717-725

53. Nakamura K, Rockson SG. Molecular targets for therapeutic lymphangiogenesis in lymphatic dysfunction and disease. Lymphat Res Biol. 2008; 6(3-4):181-189.

54. Nakamura K, Radhakrishnan K, Wong YM, Rockson SG. Anti-inflammatory pharmacotherapy with ketoprofen ameliorates experimental lymphatic vascular insufficiency in mice. PLoS One. 2009;4(12):e8380.

55. Choi I, et al. 9-cis retinoic acid promotes lymphangiogenesis and enhances lymphatic vessel regeneration: therapeutic implications of 9-cis retinoic acid for secondary lymphedema. Circulation. 2012;125(7):872-882.

56. Keim AP, et al. The multicomponent medication lymphomyosot improves the outcome of experimental lymphedema. Lymphat Res Biol. 2013;11(2):81-92. 